\title{
AFFINE-RULED VARIETIES WITHOUT THE LAURENT CANCELLATION PROPERTY
}

\author{
ADRIEN DUBOULOZ AND PIERRE-MARIE POLONI
}

\begin{abstract}
Aвstract. We describe a method to construct hypersurfaces of the complex affine $n$-space with isomorphic $\mathbb{C}^{*}$-cylinders. Among these hypersurfaces, we find new explicit counterexamples to the Laurent Cancellation Problem, i.e. hypersurfaces that are nonisomorphic, although their $\mathbb{C}^{*}$-cylinders are isomorphic as abstract algebraic varieties. We also provide examples of nonisomorphic varieties $X$ and $Y$ with isomorphic cartesian squares $X \times X$ and $Y \times Y$.
\end{abstract}

\section{INTRODUCTION}

In this paper, we consider the following cancellation problem:

Laurent Cancellation Problem. Suppose that the $\mathbb{C}^{*}$-cylinders $X \times \mathbb{C}^{*}$ and $Y \times \mathbb{C}^{*}$ over two complex affine varieties $X$ and $Y$ are isomorphic. Does it follow that $X$ and $Y$ are isomorphic as abstract algebraic varieties?

This question can of course be equivalently reformulated as the question of the uniqueness of the coefficient ring in a Laurent polynomial ring. Indeed, if we let $X=\operatorname{Spec}(A)$ and $Y=\operatorname{Spec}(B)$ for some finitely generated complex algebras, then the Laurent Cancellation Problem simply asks whether having isomorphic Laurent polynomial rings $A\left[t, t^{-1}\right] \simeq B\left[t, t^{-1}\right]$ implies that $A$ and $B$ are isomorphic themselves.

The answer is known to be positive in many cases. First of all, it is easy to see that tori have the Laurent Cancellation property, i.e. that the Laurent Cancellation Problem has a positive answer, when $X$ is isomorphic to an algebraic torus $\left(\mathbb{C}^{*}\right)^{d}$ (see e.g. Lemma 4.5 in [1]). Then, Gene Freudenburg [5] has proved that affine curves (i.e. complex affine algebraic varieties $X$ of dimension 1) have also the Laurent Cancellation property. Moreover, using ideas similar to that for Iitaka and Fujita's strong cancellation theorem [7], the first author provided in [3] an affirmative answer for large classes of varieties. In particular, Laurent Cancellation does hold if $X$ is of log-general type [3, Proposition 2] or if $X$ is a smooth factorial affine surface with logarithmic Kodaira dimension different from 1 (see [3, Proposition 12]). On the other hand, counterexamples were given in [3] in the form of pairs of nonisomorphic smooth factorial affine varieties $X$ and $Y$ of dimension $d \geq 2$ and logarithmic Kodaira dimension $d-1$ such that $X \times \mathbb{C}^{*}$ and $Y \times \mathbb{C}^{*}$ are isomorphic [3, Propositions 6 and 9].

The main purpose of the present paper is to provide a general method to construct explicit counterexamples to the Laurent Cancellation Problem. All these examples will be realized as hypersurfaces of affine space $\mathbb{A}^{n+1}$ that are defined by an equation of the form $t^{\ell} f\left(x_{1}, \ldots, x_{n}\right)=1$, where $f$ is a regular function which is semi-invariant for some action of the algebraic multiplicative group $\mathbb{G}_{m}$ on $\mathbb{A}^{n}=\operatorname{Spec}\left(\mathbb{C}\left[x_{1}, \ldots, x_{n}\right]\right)$. We will show that some of the examples in [3] can actually be reinterpreted as being particular cases of our construction, and will also obtain new examples of varieties that fail the Laurent Cancellation property, notably affine algebraic varieties of negative logarithmic Kodaira dimension.

As a byproduct of our construction, we will also get explicit examples illustrating the following result.

Theorem. There exist smooth affine algebraic varieties (of every dimension $d \geq 2$ ) $X$ and $Y$ which are nonisomorphic, although their cartesian product with themselves, $X \times X$ and $Y \times Y$, are isomorphic.

The paper is organized as follows:

In the first section, we study hypersurfaces $\widetilde{X}_{f, \ell}$ of $\mathbb{C}^{n+1}$ that are defined by the equation $t^{\ell} f=1$, where $f \in \mathbb{C}\left[x_{1}, \ldots, x_{n}\right]$ is a polynomial which is semi-invariant for an effective algebraic action of $\mathbb{C}^{*}$ on $\mathbb{C}^{n}$. We

2010 Mathematics Subject Classification. 14R05, 14R25, 14L30.

Key words and phrases. Laurent Cancellation Problem; hypersurfaces; affine-ruled varieties.

This research was supported in part by the ANR Grant BirPol ANR-11-JS01-004-01. The second author was supported by the Universität Basel Forschungsfonds. 
establish sufficient conditions under which the $\mathbb{C}^{*}$-cylinders $\tilde{X}_{f, \ell} \times \mathbb{C}^{*}$ and $\tilde{X}_{f, \ell^{\prime}} \times \mathbb{C}^{*}$ are isomorphic. We also develop a general strategy to prove that two given $\widetilde{X}_{f, \ell}$ and $\widetilde{X}_{f, \ell^{\prime}}$ are not isomorphic.

The second section is devoted to the case of surfaces $\widetilde{X}_{f, \ell}$ where $f \in \mathbb{C}[x, y]$ is of the form $f=x^{p}+y^{q}$. Our main result is the following.

Theorem. Let $p \geq 2$ be a prime number, let $q \geq 3$ be an integer relatively prime with $p$ and let $\ell \geq 2$ be relatively prime with $m=p q$. Then, the smooth factorial affine surfaces of respective equation $t\left(x^{p}+y^{q}\right)=1$ and $t^{\ell}\left(x^{p}+y^{q}\right)=1$ are not isomorphic, although they have isomorphic $\mathbb{C}^{*}$-cylinders.

In Section 3, we focus on the case of varieties of negative logarithmic Kodaira dimension. We first improve a result of [3] by showing that Laurent Cancellation does hold for all smooth affine surfaces of negative logarithmic Kodaira dimension, and then construct explicit higher dimensional examples that fail Laurent Cancellation.

Finally, examples of nonisomorphic affine varieties with isomorphic squares are given in Section 4 .

Acknowledgments. We are grateful to Jérémy Blanc and Jean-Philippe Furter for their helpful suggestions about Proposition 23.

\section{Semi-invariants of $\mathbb{G}_{m}$-ACTions and $\mathbb{A}_{*}^{1}$-Cylinders}

Unless otherwise specified, we will work over the field $\mathbb{C}$ of complex numbers. We will denote by $\mathbb{A}^{n}=$ $\mathbb{A}_{\mathbb{C}}^{n}=\operatorname{Spec}\left(\mathbb{C}\left[x_{1}, \ldots, x_{n}\right]\right)$ the affine $n$-space and by $\mathbb{A}_{*}^{1}=\mathbb{A}^{1} \backslash\{0\}=\operatorname{Spec}\left(\mathbb{C}\left[t, t^{-1}\right]\right)$ the affine line minus the origin.

1.1. Isotrivial fibrations associated to semi-invariant regular functions. Let $X$ be a complex affine variety endowed with an effective action $\mu: \mathbb{G}_{m} \times X \rightarrow X$ of the multiplicative group $\mathbb{G}_{m}=\mathbb{G}_{m}, \mathbb{C}=$ $\operatorname{Spec}\left(\mathbb{C}\left[t^{ \pm 1}\right]\right)$. Its coordinate $\operatorname{ring} A=\mathcal{O}(X)$ is then equipped with a natural $\mathbb{Z}$-grading by the subspaces $A_{m}=\left\{f \in A, f(\mu(t, x))=t^{m} f(x) \forall x \in X\right\}$ of semi-invariants of weight $m \in \mathbb{Z}$.

Notation 1. Given a semi-invariant $f$ of weight $m \neq 0$, we denote by $X_{f}$ the principal open subset of $X$ where $f$ does not vanish and, for every $\ell \geq 1$, by $\tilde{X}_{f, \ell}$ the closed subvariety of $X \times \mathbb{G}_{m}=\operatorname{Spec}\left(A\left[t^{ \pm 1}\right]\right)$ defined by the equation $t^{\ell} f=1$. Note that $\widetilde{X}_{f, \ell}$ can also be seen as the closed subvariety of $X \times \mathbb{A}^{1}$ given by the same equation $t^{\ell} f=1$.

The restriction to $\tilde{X}_{f, \ell}$ of the first projection $\operatorname{pr}_{X}$ is an étale Galois cover $\tilde{X}_{f, \ell} \rightarrow X_{f} \simeq \widetilde{X}_{f, 1}$ with Galois group $\mathbb{Z}_{\ell}$. On the other hand, the second projection $\operatorname{pr}_{\mathbb{G}_{m}}$ restricts on $\tilde{X}_{f, \ell}$ to an isotrivial fibration $\rho_{f, \ell}: \widetilde{X}_{f, \ell} \rightarrow \mathbb{G}_{m}$ with fiber $F=f^{-1}(1)=\operatorname{Spec}(A /(f-1))$, which becomes trivial after the finite étale base change $\psi: C=\operatorname{Spec}\left(\mathbb{C}\left[v^{ \pm 1}\right]\right) \rightarrow \mathbb{G}_{m}, v \mapsto v^{m}$. Indeed, since $f$ is a semi-invariant of weight $m$, the morphism

$$
F \times C \rightarrow \widetilde{X}_{f, \ell} \times_{\mathbb{G}_{m}} C,(x, v) \mapsto\left(\left(\mu\left(v^{-\ell}, x\right), v^{m}\right), v\right)
$$

is an isomorphism of schemes over $C$.

Proposition 2. With the notation above, the following hold:

(1) If $\ell^{\prime}$ is congruent to $\ell$ or $-\ell$ modulo $m$, then the fibrations $\rho_{f, \ell}: \widetilde{X}_{f, \ell} \rightarrow \mathbb{G}_{m}$ and $\rho_{f, \ell^{\prime}}: \widetilde{X}_{f, \ell^{\prime}} \rightarrow \mathbb{G}_{m}$ are isomorphic up to an automorphism of $\mathbb{G}_{m}$.

(2) If the residue classes modulo $m$ of $\ell$ and $\ell^{\prime}$ generate the same subgroup of $\mathbb{Z}_{m}$, i.e. if $\operatorname{gcd}(\ell, m)=$ $\operatorname{gcd}\left(\ell^{\prime}, m\right)$, then $\tilde{X}_{f, \ell} \times \mathbb{A}_{*}^{1}$ and $\tilde{X}_{f, \ell^{\prime}} \times \mathbb{A}_{*}^{1}$ are isomorphic as abstract algebraic varieties.

Proof. Indeed, if $\ell= \pm \ell^{\prime}+k m$ for some $k \in \mathbb{Z}$, then the morphism $\Phi: \widetilde{X}_{f, \ell} \rightarrow \widetilde{X}_{f, \ell^{\prime}},(x, t) \mapsto\left(\mu\left(t^{k}, x\right), t^{ \pm 1}\right)$ is an isomorphism for which we have a commutative diagram



For the second assertion, let us identify $\widetilde{X}_{f, \ell} \times \mathbb{A}_{*}^{1}$ and $\widetilde{X}_{f, \ell^{\prime}} \times \mathbb{A}_{*}^{1}$ with the closed subvarieties of $X \times\left(\mathbb{A}_{*}^{1}\right)^{2}=$ $\operatorname{Spec}\left(A\left[t^{ \pm 1}, u^{ \pm 1}\right]\right)$ defined by the equations $t^{\ell} f-1=0$ and $t^{\ell^{\prime}} f-1=0$, respectively. Let $d=\operatorname{gcd}(\ell, m)=$ 
$\operatorname{gcd}\left(\ell^{\prime}, m\right)$. Then, there exist integers $a, b \in \mathbb{Z}$ satisfying $\ell^{\prime}=a \ell+b m$ such that $a$ is coprime with $\frac{m}{d}$. This guarantees in turn the existence of a matrix

$$
A=\left(\begin{array}{cc}
a & \frac{m}{d} \\
\alpha & \beta
\end{array}\right) \in \mathrm{SL}_{2}(\mathbb{Z})
$$

corresponding to an automorphism $\sigma(t, u)=\left(t^{a} u^{m / d}, t^{\alpha} u^{\beta}\right)$ of the torus $\left(\mathbb{A}_{*}^{1}\right)^{2}=\operatorname{Spec}\left(\mathbb{C}\left[t^{ \pm 1}, u^{ \pm 1}\right]\right)$. Finally, a straightforward computation shows that the automorphism of $X \times\left(\mathbb{A}_{*}^{1}\right)^{2}$ defined by $(x, t, u) \mapsto$ $\left(\mu\left(t^{b} u^{-\ell / d}, x\right), \sigma(t, u)\right)$ maps $\widetilde{X}_{f, \ell^{\prime}} \times \mathbb{A}_{*}^{1}$ isomorphically onto $\widetilde{X}_{f, \ell} \times \mathbb{A}_{*}^{1}$.

Remark 3. Note that the isomorphism constructed in the proof of Proposition 2 does not preserve the induced isotrivial fibration $\rho_{f, \ell} \circ \mathrm{pr}_{1}: \widetilde{X}_{f, \ell} \times \mathbb{A}_{*}^{1} \rightarrow \mathbb{G}_{m}$ with fiber $F \times \mathbb{A}_{*}^{1}$.

In particular, observe that Proposition 2 implies that if $f$ is a semi-invariant regular function of weight $m \neq 0$, then the varieties $\widetilde{X}_{f, \ell} \times \mathbb{A}_{*}^{1}$ are isomorphic to $\widetilde{X}_{f, 1} \times \mathbb{A}_{*}^{1}=X_{f} \times \mathbb{A}_{*}^{1}$ for all integers $\ell \geq 1$ relatively prime with $m$. Let us list some consequences of this observation.

Corollary 4. Suppose that the variety $X_{f}$ is not $\mathbb{A}_{*}^{1}$-uniruled (for instance, that $X_{f}$ is smooth of log-general type). Then for all $\ell \in \mathbb{Z}_{\geq 1}$ relatively prime with $m$ the varieties $\tilde{X}_{f, \ell}$ are isomorphic.

Proof. Indeed, by a strong cancellation theorem due to Iitaka and Fujita [7] (see also [3]), every isomorphism between $\widetilde{X}_{f, \ell} \times \mathbb{A}_{*}^{1}$ and $\widetilde{X}_{f, \ell^{\prime}} \times \mathbb{A}_{*}^{1}$ descends to an isomorphism between $\widetilde{X}_{f, \ell}$ and $\widetilde{X}_{f, \ell^{\prime}}$.

Corollary 5. Suppose that $X_{f}$ is a smooth factorial affine surface of logarithmic Kodaira dimension $\bar{\kappa}\left(X_{f}\right)$ different from 1 . Then the surfaces $\widetilde{X}_{f, \ell}$ are isomorphic for all $\ell \geq 1$ relatively prime with $m$.

Proof. Since $\tilde{X}_{f, \ell} \times \mathbb{A}_{*}^{1}$ is isomorphic to $X_{f} \times \mathbb{A}_{*}^{1}$ for all $\ell \in \mathbb{Z}_{\geq 1}$ relatively prime with $m$, we deduce that $\widetilde{X}_{f, \ell}$ is smooth, factorial, of the same logarithmic Kodaira dimension as $X_{f}$. So the assertion follows from Proposition 12 in [3] which asserts that $\mathbb{A}_{*}^{1}$-cancellation holds for smooth factorial affine surfaces of logarithmic Kodaira dimension different from 1.

1.2. Semi-invariant hypersurfaces of affine spaces. In this subsection, we consider more specifically the case of principal open subsets $X_{f} \subset \mathbb{A}^{n}$ associated to semi-invariant polynomials $f \in \mathbb{C}\left[x_{1}, \ldots, x_{n}\right]$. As a third application of Proposition 2, we determine the groups $\mathcal{O}\left(\widetilde{X}_{f, \ell}\right)^{*}$ of invertible regular functions on the varieties $\widetilde{X}_{f, \ell}$ when $f$ is irreducible and $\ell$ is coprime with $m$.

Lemma 6. Let $n, m, \ell \geq 1$ be integers, let $f \in \mathbb{C}\left[x_{1}, \ldots, x_{n}\right]$ be semi-invariant of weight $m \geq 1$ under an effective regular action of the multiplicative group $\mathbb{G}_{m}$ on $\mathbb{C}\left[x_{1}, \ldots, x_{n}\right]$ and let $\widetilde{X}_{f, \ell} \subset \mathbb{A}^{n} \times \mathbb{A}_{*}^{1}=$ $\operatorname{Spec}\left(\mathbb{C}\left[x_{1}, \ldots, x_{n}\right]\left[t^{ \pm 1}\right]\right)$ be the hypersurface defined by the equation $t^{\ell} f=1$. If $f$ is irreducible and $\ell$ and $m$ are coprime, then

$$
\mathcal{O}\left(\widetilde{X}_{f, \ell}\right)^{*}=\left\{\lambda t^{i} \mid \lambda \in \mathbb{C}^{*}, i \in \mathbb{Z}\right\} .
$$

Proof. First note that since the polynomial $f \in \mathbb{C}\left[x_{1}, \ldots, x_{n}\right]$ is irreducible, we have

$$
\mathcal{O}\left(\widetilde{X}_{f, 1}\right)^{*}=\left(\mathbb{C}\left[x_{1}, \ldots, x_{n}\right] /(t f-1)\right)^{\times}=\left\{\lambda t^{i} \mid \lambda \in \mathbb{C}^{*}, i \in \mathbb{Z}\right\} \simeq \mathbb{C}^{*} \cdot \mathbb{Z} .
$$

By Proposition 2, if $\ell$ is coprime with $m$, then the varieties $\widetilde{X}_{f, \ell} \times \mathbb{A}_{*}^{1}$ and $\widetilde{X}_{f, 1} \times \mathbb{A}_{*}^{1}$ are isomorphic and have thus isomorphic unit groups. On the other hand, denoting $\mathcal{O}\left(X \times \mathbb{A}_{*}^{1}\right)=\mathcal{O}(X)\left[u, u^{-1}\right]$, we have that $\mathcal{O}\left(X \times \mathbb{A}_{*}^{1}\right)^{*}=\left\{a \cdot u^{i} \mid a \in \mathcal{O}(X)^{*}, i \in \mathbb{Z}\right\} \simeq \mathcal{O}(X)^{*} \cdot \mathbb{Z}$ for every affine algebraic variety $X$. Therefore, we get that $\mathcal{O}\left(\widetilde{X}_{f, \ell} \times \mathbb{A}_{*}^{1}\right)^{*} / \mathbb{C}^{*} \simeq \mathcal{O}\left(\widetilde{X}_{f, 1} \times \mathbb{A}_{*}^{1}\right)^{*} / \mathbb{C}^{*} \simeq \mathbb{Z}^{2}$ if $\ell$ is coprime with $m$, and the lemma follows.

Remark 7. We believe that one can drop the hypothesis $\operatorname{gcd}(m, \ell)=1$ in the previous lemma and that the equality $\mathcal{O}\left(\widetilde{X}_{f, \ell}\right)^{*}=\left\{\lambda t^{i} \mid \lambda \in \mathbb{C}^{*}, i \in \mathbb{Z}\right\}$ holds for all $\ell \geq 1$. Nevertheless, this seems to be a difficult question (see e.g. Conjecture 2.9 in [4]).

The previous lemma turns out to be a useful tool to decide when a variety $\widetilde{X}_{f, \ell}$ is nonisomorphic to $\widetilde{X}_{f, 1}$, since it allows us to reduce the problem to the study of the generic fibers of the projections $\rho_{f, \ell}: \widetilde{X}_{f, \ell} \rightarrow \mathbb{A}_{*}^{1}$ for all $\ell$ coprime with $m$. Namely, we have the following result: 
Proposition 8. Let $f \in \mathbb{C}\left[x_{1}, \ldots, x_{n}\right]$ be irreducible and semi-invariant of weight $m \geq 1$ under an effective regular action $\mu$ of the multiplicative group $\mathbb{G}_{m}$ on $\mathbb{C}\left[x_{1}, \ldots, x_{n}\right]$. Let $\mathbf{k}=\mathbb{C}(t)$ and denote, for every $\ell \geq 1$, by $Y_{\ell}$ the closed subvariety of $\mathbb{A}_{\mathbf{k}}^{n}$ defined by the equation $f=t^{-\ell}$. Suppose that the varieties $\widetilde{X}_{f, \ell}$ and $\widetilde{\widetilde{X}}_{f, \ell^{\prime}}$ are isomorphic for some integers $\ell, \ell^{\prime}$ both coprime with $m$. Let further $\ell^{\prime \prime} \geq 1$ be congruent to $-\ell^{\prime}$ modulo m. Then, $Y_{\ell}$ is isomorphic to $Y_{\ell^{\prime}}$ or to $Y_{\ell^{\prime \prime}}$.

Proof. By Lemma 6 , the groups $\mathcal{O}\left(\widetilde{X}_{f, \ell}\right)^{*} / \mathbb{C}^{*}$ and $\mathcal{O}\left(\widetilde{X}_{f, \ell^{\prime}}\right)^{*} / \mathbb{C}^{*}$ are both isomorphic to $\mathbb{Z}$, generated by the image of $t$. So every isomorphism $\Phi: \widetilde{X}_{f, \ell} \rightarrow \widetilde{X}_{f, \ell^{\prime}}$ induces an automorphism $\varphi$ of $\mathbb{A}_{*}^{1}=\operatorname{Spec}\left(\mathbb{C}\left[t^{ \pm 1}\right]\right)$ of the form $t \mapsto a t^{ \pm 1}$ for some $a \in \mathbb{C}^{*}$. Composing $\Phi$ with the automorphism of $\widetilde{X}_{f, \ell}$ defined by $(x, t) \mapsto$ $\left(\mu\left(a^{\ell / m}, x\right), a^{-1} t\right)$, where $a^{\ell / m}$ denotes a $m$-th root of $a^{\ell}$, we get an isomorphism $\Phi_{2}: \widetilde{X}_{f, \ell} \rightarrow \widetilde{X}_{f, \ell^{\prime}}$ which fits into a commutative diagram

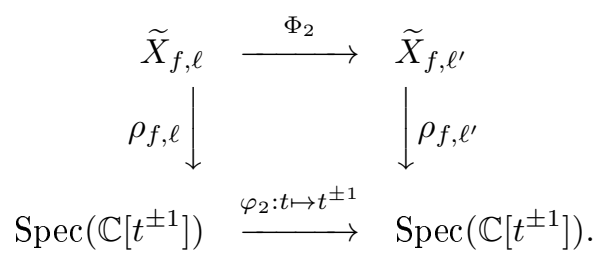

If $\varphi_{2}(t)=t$, then we get an isomorphism between the generic fibers of $\rho_{f, \ell}$ and $\rho_{f, \ell^{\prime}}$, which are isomorphic to $Y_{\ell}$ and $Y_{\ell^{\prime}}$, respectively. If $\varphi_{2}(t)=t^{-1}$, then composing $\Phi_{2}$ further with the isomorphism between $\widetilde{X}_{f, \ell^{\prime}}$ and $\widetilde{X}_{f, \ell^{\prime \prime}}$ defined by $(x, t) \mapsto\left(\mu\left(t^{q}, x\right), t^{-1}\right)$, where $q \geq 1$ satisfies the equality $\ell^{\prime \prime}=-\ell^{\prime}+q m$, we get an isomorphism $\Phi_{3}: \widetilde{X}_{f, \ell} \rightarrow \widetilde{X}_{f, \ell^{\prime \prime}}$ which fits into a commutative diagram

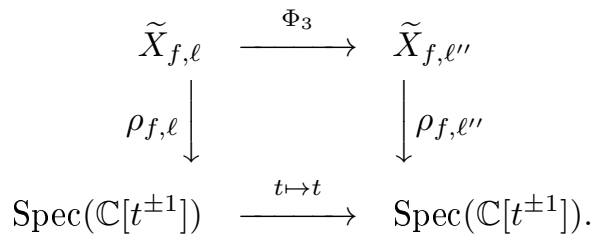

This concludes the proof, since the above diagram implies that $Y_{\ell}$ and $Y_{\ell^{\prime \prime}}$ are isomorphic.

\section{Factorial affine surfaces failing Laurent Cancellation}

As a first application of the previous techniques, we present new explicit examples of factorial surfaces failing Laurent cancellation. They are all realized as hypersurfaces $\widetilde{X}_{f, \ell}$ for irreducible polynomials $f \in \mathbb{C}[x, y]$ which are semi-invariant under a faithful linear $\mathbb{G}_{m}$-action on $\mathbb{A}^{2}=\operatorname{Spec}(\mathbb{C}[x, y])$ with positive weights. More precisely, given positive integers $p, q \geq 1$, we consider the polynomial $f=x^{p}+y^{q}$ which is semi-invariant of weight $m=p q$ under the action $\mu: \mathbb{G}_{m} \times \mathbb{A}^{2} \rightarrow \mathbb{A}^{2}$ defined by $\mu(\lambda,(x, y))=\left(\lambda^{q} x, \lambda^{p} y\right)$.

Note that if $p$ or $q$ is equal to 1 , then the complements $X_{f}$ of the zero loci of polynomials $f$ are isomorphic to $\mathbb{A}^{1} \times \mathbb{A}_{*}^{1}$, and so are all the associated surfaces $\widetilde{X}_{f, \ell}, \ell \in\{1, \ldots, m\}$. Therefore, let $p, q$ be both greater than 1 , and relatively prime. Then $X_{f}$ is isomorphic to the product of the smooth affine Fermat type curve $C=$ $\left\{x^{p}+y^{q}=1\right\} \subset \mathbb{A}^{2}$ with $\mathbb{A}_{*}^{1}$. Recall that a smooth projective model $\bar{C}$ of $C$ has genus $g(\bar{C})=\frac{(p-1)(q-1)}{2}>0$. Consequently, the logarithmic Kodaira dimension of $\widetilde{X}_{f, 1} \simeq X_{f}$ is equal to 1 . Since the logarithmic Kodaira dimension is invariant under finite étale covers, all associated surfaces $\widetilde{X}_{f, \ell}$ are also of logarithmic Kodaira dimension 1.

All surfaces $X_{f}$ obtained in this way are factorial and the associated surfaces $\tilde{X}_{f, \ell}$ are $\mathbb{A}_{*}^{1}$-uniruled. They are in fact canonically $\mathbb{A}_{*}^{1}$-ruled over $\mathbb{A}^{1}$ by the restriction $q_{\ell}: \widetilde{X}_{f, \ell} \rightarrow \mathbb{A}^{1}$ of the rational map $V_{\ell} \rightarrow \mathbb{P}^{1}$ defined by the mobile part of the divisor $K_{V_{\ell}}+B_{\ell}$ on a smooth projective completion $V_{\ell}$ of $\widetilde{X}_{f, \ell}$ with reduced SNC boundary $B_{\ell}$ (see e.g. [8, Chapter 2, Section 6]). In view of Corollaries 4 and 5 we thus expect to find counterexamples to the Laurent Cancellation Problem among these surfaces.

The first case to consider is the case $(p, q)=(2,3)$. Nevertheless, it turns out to be deceptive. Indeed, on the one hand, Proposition 2 implies that every surface $\widetilde{X}_{f, \ell}$ with $f=x^{2}+y^{3}$ is isomorphic to $\widetilde{X}_{f, 1}$, to $\widetilde{X}_{f, 2}$ or to $\widetilde{X}_{f, 3}$. On the other hand, we have the following result. 
Lemma 9. Let $f=x^{2}+y^{3} \in \mathbb{C}[x, y], n \geq 1$, and let $\ell, \ell^{\prime} \in\{1,2,3\}$ be distinct. Then for every $n \geq 0$, the varieties $\widetilde{X}_{f, \ell} \times\left(\mathbb{A}_{*}^{1}\right)^{n}$ and $\widetilde{X}_{f, \ell^{\prime}} \times\left(\mathbb{A}_{*}^{1}\right)^{n}$ are not isomorphic.

Proof. Let us identify for each $i \in\{1,2,3\}$ the surface $\widetilde{X}_{f, i}$ with the closed subvariety of $\operatorname{Spec}\left(\mathbb{C}\left[x, y, t^{ \pm 1}\right]\right)$ defined by the equation, $t^{i}\left(x^{2}+y^{3}\right)=1$. The log-canonical $\mathbb{A}_{*}^{1}$-fibration $q_{i}: \widetilde{X}_{f, i} \rightarrow \mathbb{A}^{1}$ of $\widetilde{X}_{f, i}$ coincides with the algebraic quotient morphism of the $\mathbb{G}_{m}$-action $\lambda \cdot(x, y, t)=\left(\lambda^{3} x, \lambda^{2} y, \lambda^{-6 / i} t\right)$. More concretely, we have

$$
\begin{cases}q_{1}: \widetilde{X}_{f, 1} \rightarrow \mathbb{A}^{1}, & (x, y, v) \mapsto x^{2} t, \\ q_{2}: \widetilde{X}_{f, 2} \rightarrow \mathbb{A}^{1}, & (x, y, v) \mapsto x t, \\ q_{3}: \widetilde{X}_{f, 3} \rightarrow \mathbb{A}^{1}, & (x, y, v) \mapsto y t .\end{cases}
$$

Furthermore, since $\kappa\left(\widetilde{X}_{f, i} \times\left(\mathbb{A}_{*}^{1}\right)^{n}\right)=\kappa\left(\widetilde{X}_{f, i}\right)$, the log-canonical fibration of $\widetilde{X}_{f, i} \times\left(\mathbb{A}_{*}^{1}\right)^{n}$ coincides with $\tau_{i}=q_{i} \circ \operatorname{pr}_{\tilde{X}_{f, i}}: \widetilde{X}_{f, i} \times\left(\mathbb{A}_{*}^{1}\right)^{n} \rightarrow \mathbb{A}^{1}$. It is straightforward to check that the $\mathbb{A}_{*}^{1}$-fibrations $q_{i}$, hence the $\left(\mathbb{A}_{*}^{1}\right)^{n+1}$-fibrations $\tau_{i}$, have different types of degenerate fibers: all their fibers are isomorphic to $\mathbb{A}_{*}^{1}$ when equipped with their reduced structures, but $q_{1}$ has two multiple fibers of multiplicities 2 and 3, respectively, $q_{2}$ has two multiple fibers of multiplicity 3 while $q_{3}$ has three multiple fibers of multiplicity 2 . The lemma follows then, since an isomorphism $\widetilde{X}_{f, i} \times\left(\mathbb{A}_{*}^{1}\right)^{n}$ and $\tilde{X}_{f, j} \times\left(\mathbb{A}_{*}^{1}\right)^{n}, i, j=1,2,3$, must be compatible with these log-canonical fibrations.

The next simplest case, namely the case $(p, q)=(2,5)$, does lead to counterexamples.

Proposition 10. The hypersurfaces $X_{1}, X_{3} \subset \mathbb{A}^{3}=\operatorname{Spec}(\mathbb{C}[x, y, t])$ defined by the equation $t\left(x^{2}+y^{5}\right)=1$ and $t^{3}\left(x^{2}+y^{5}\right)=1$, respectively, are counterexamples to the Laurent Cancellation Problem.

Proof. Consider the polynomial $f=x^{2}+y^{5} \in \mathbb{C}[x, y]$ and observe that $X_{1}$ and $X_{3}$ are isomorphic to the hypersurfaces $\widetilde{X}_{f, 1}$ and $\widetilde{X}_{f, 3}$, respectively. Since $f$ is semi-invariant of weight 10 for the linear $\mathbb{G}_{m}$-action on $\mathbb{A}^{2}$ with weights $(5,2)$, the $\mathbb{A}_{*}^{1}$-cylinders $X_{1} \times \mathbb{A}_{*}^{1}$ and $X_{3} \times \mathbb{A}_{*}^{1}$ are isomorphic by Proposition 2. To show that $X_{1}$ and $X_{3}$ are not isomorphic, it is enough, by virtue of Proposition 8, to check that the curve $Y_{1} \subset \mathbb{A}_{\mathbb{C}(t)}^{2}=\operatorname{Spec}(\mathbb{C}(t)[x, y])$ given by the equation $x^{2}+y^{5}=t^{-1}$ is isomorphic over $\mathbb{C}(t)$ neither to the curve $Y_{3}$ of equation $x^{2}+y^{5}=t^{-3}$, nor to that $Y_{7}$ of equation $x^{2}+y^{5}=t^{-7}$. This can be seen using elementary geometric arguments as follows.

A $\mathbb{C}(t)$-isomorphism $\psi: Y_{1} \stackrel{\sim}{\rightarrow} Y_{i}$ between $Y_{1}$ and $Y_{i}, i=3,7$, must (if it exists) extend to an isomorphism $\Psi: \bar{Y}_{1} \stackrel{\sim}{\rightarrow} \bar{Y}_{i}$ between the respective normalizations $\bar{Y}_{1}$ and $\bar{Y}_{i}$ of the projective closures of $Y_{1}$ and $Y_{i}$ in $\mathbb{P}_{\mathbb{C}(t)}^{2}=\operatorname{Proj}(\mathbb{C}(t)[x, y, z])$. A direct computation shows that all these curves have genus 2 and that their respective canonical degree 2 covers $\bar{\pi}_{i}: \bar{Y}_{j} \rightarrow \mathbb{P}_{\mathbb{C}(v)}^{1}$ defined by the complete linear systems $\left|K_{\bar{Y}_{j}}\right|, j=1,3,7$, coincide with the maps induced by the projection from the point $[1: 0: 0]$ in $\mathbb{P}_{\mathbb{C}(t)}^{2}$. The restriction of $\bar{\pi}_{i}$ to $Y_{i}$ coincides with the projection $\pi_{i}=\operatorname{pr}_{y}: Y_{i}=\left\{x^{2}+y^{5}-t^{-i}=0\right\} \rightarrow \mathbb{A}_{\mathbb{C}(t)}^{1}=\operatorname{Spec}(\mathbb{C}(t)[y])$, and since $\Psi^{*} K_{\bar{Y}_{i}}=K_{\bar{Y}_{1}}$, it follows that there exists a $\mathbb{C}(t)$-automorphism $\theta$ of $\mathbb{A}_{\mathbb{C}(t)}^{1}$ for which the following diagram commutes

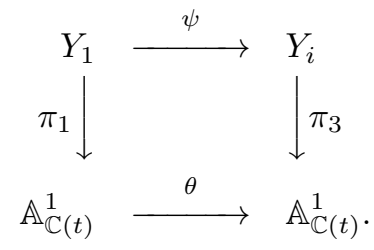

So $\theta$ is an affine transformation of the form $y \mapsto a(t) y+b(t)$ for some pair $(a(t), b(t)) \in \mathbb{C}(t)^{*} \times \mathbb{C}(t)$ which maps the branch locus $B_{1}=\left\{y^{5}-t^{-1}=0\right\}$ of $\pi_{1}$ isomorphically onto that $B_{i}=\left\{y^{5}-t^{-i}=0\right\}$ of $\pi_{i}$, $i=3,7$. Thus $b(t)=0$ necessarily and $a(t)^{5}=t^{-i+1}$, which is absurd since neither $t^{-2}$ nor $t^{-6}$ admits a fifth root in $\mathbb{C}(t)$. This shows that $X_{1}$ and $X_{3}$ are not isomorphic.

Remark 11. The above surfaces $X_{1}$ and $X_{3}$ are precisely those constructed in a more geometric fashion in [3, Subsection 2.2]. This can be seen by considering the structure of their log-canonical $\mathbb{A}_{*}^{1}$-fibrations. With the same notation as in the proof of Proposition 10, these fibrations coincide with the morphisms

$$
q_{1}: X_{1} \rightarrow \mathbb{A}^{1},(x, y, t) \mapsto y^{5} t \quad \text { and } \quad q_{3}: X_{3} \rightarrow \mathbb{A}^{1},(x, y, t) \mapsto y^{5} t^{3} .
$$


Note that for the same reason as in the proof of Lemma 9 , every isomorphism $\Psi$ between $X_{3} \times \mathbb{A}_{*}^{1}$ and $X_{1} \times \mathbb{A}_{*}^{1}$ must be compatible with the log-canonical $\left(\mathbb{A}_{*}^{1}\right)^{2}$-fibrations $q_{1} \circ \operatorname{pr}_{1}: X_{1} \times \mathbb{A}_{*}^{1} \rightarrow \mathbb{A}^{1}$ and $q_{3} \circ \operatorname{pr}_{1}: X_{3} \times \mathbb{A}_{*}^{1} \rightarrow \mathbb{A}^{1}$.

This does of course hold for the explicit isomorphism $\Psi: X_{3} \times \mathbb{A}_{*}^{1} \stackrel{\sim}{\rightarrow} X_{1} \times \mathbb{A}_{*}^{1}$ constructed via the procedure described in the proof of Proposition 2, which is obtained as follows. With the notation of this proof, we have in our case $\ell=1, \ell^{\prime}=3, m=10, d=1$, and we can take $a=3, b=0, \alpha=-1$ and $\beta=-3$. This gives the isomorphism $\Psi:(x, y, t, u) \mapsto\left(u^{-5} x, u^{-2} y, t^{3} u^{10}, t^{-1} u^{-3}\right)$. One thus has $\Psi^{*}\left(y^{5} t\right)=\left(u^{-10} y^{5}\right)\left(t^{3} u^{10}\right)=y^{5} t^{3}$, hence a commutative diagram

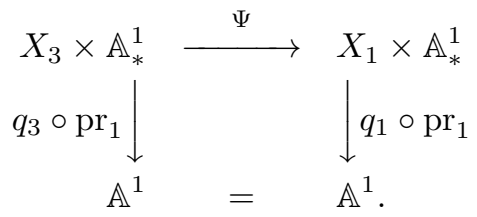

Let us emphasize two ingredients which played a crucial role in the proof of the fact that the above surfaces $X_{1}$ and $X_{3}$ are not isomorphic. The first one is that the curves $Y_{i}, i=1,3,7$, are cyclic covers of $\mathbb{A}_{\mathbb{C}(t)}^{1}$. The second one is that every isomorphism between them descends to an automorphism of $\mathbb{A}_{\mathbb{C}(t)}^{1}$. Generalizing this type of arguments allows us to obtain the following infinite families of counterexamples.

Theorem 12. Let $p \geq 2$ be a prime number, let $q \geq 3$ be an integer relatively prime with $p$ and let $f=x^{p}+y^{q} \in \mathbb{C}[x, y]$. Then for every $\ell \geq 2$ relatively prime with $m=p q$, the surfaces $\widetilde{X}_{f, 1}$ and $\widetilde{X}_{f, \ell}$ are nonisomorphic, with isomorphic $\mathbb{A}_{*}^{1}$-cylinders.

Proof. The polynomial $f$ being an irreducible semi-invariant of weight $m$ for the linear action of $\mathbb{G}_{m}$ on $\mathbb{A}^{2}$ with weight $(q, p)$, the fact that $X_{1}=\widetilde{X}_{f, 1}$ and $X_{\ell}=\tilde{X}_{f, \ell}$ have isomorphic $\mathbb{A}_{*}^{1}$-cylinders follows again from Proposition 2 .

On the other hand, by virtue of Proposition $8, X_{1}$ and $X_{\ell}$ are nonisomorphic provided that the curve $Y_{1}=\left\{x^{p}+y^{q}-t^{-1}=0\right\}$ in $\mathbb{A}_{\mathbb{C}(t)}^{2}$ is isomorphic over $\mathbb{C}(t)$ neither to $Y_{\ell}=\left\{x^{p}+y^{q}-t^{-\ell}=0\right\}$ nor to $Y_{\ell^{\prime \prime}}=\left\{x^{p}+y^{q}-t^{-\ell^{\prime \prime}}=0\right\}$ for some integer $\ell^{\prime \prime} \geq 1$ congruent to $-\ell^{\prime}$ modulo $m$. This can be proved in exactly the same way as for Proposition 10 as soon as we show that, if it exists, a $\mathbb{C}(t)$-isomorphism $\psi: Y_{1} \stackrel{\sim}{\rightarrow} Y_{j}$, $j=\ell, \ell^{\prime \prime}$ descends to a $\mathbb{C}(t)$-automorphism $\theta$ of $\mathbb{A}_{\mathbb{C}(t)}^{1}$ making the following diagram commutative

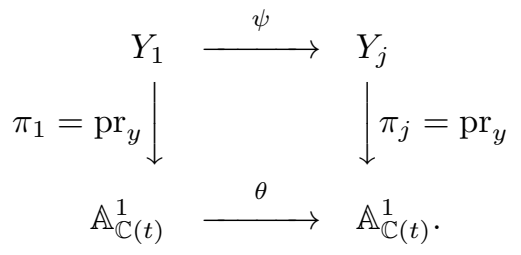

Again, every $\mathbb{C}(t)$-isomorphism $\psi: Y_{1} \stackrel{\sim}{\rightarrow} Y_{j}$ uniquely extends to a $\mathbb{C}(t)$-isomorphism $\Psi: \bar{Y}_{1} \stackrel{\sim}{\rightarrow} \bar{Y}_{j}$ between the normalizations $\bar{Y}_{i}$ of the respective projective closures of the curves $Y_{i}$ in $\mathbb{P}_{\mathbb{C}(t)}^{2}$. The latter are smooth curves of genus $g=\frac{(p-1)(q-1)}{2} \geq 2$ on which $\pi_{i}$ extends to a cyclic Galois cover $\bar{\pi}_{i}: \bar{Y}_{i} \rightarrow \mathbb{P}_{\mathbb{C}(t)}^{1}$ of prime order $p$, which is totally ramified over the $\mathbb{C}(t)$-rational point $\mathbb{P}_{\mathbb{C}(t)}^{1} \backslash \mathbb{A}_{\mathbb{C}(t)}^{1}$. Base changing to the algebraic closure $K$ of $\mathbb{C}(t)$, it follows from [6, Theorem 1] and [10, Main Theorem], which are actually stated for complex curves but whose proofs carry on verbatim to smooth curves defined over an algebraically closed field of characteristic zero, that $\Psi_{K}: \bar{Y}_{1, K} \stackrel{\sim}{\rightarrow} \bar{Y}_{j, K}$ descends to a $K$-automorphism $\bar{\theta}_{K}: \mathbb{P}_{K}^{1} \stackrel{\sim}{\rightarrow} \mathbb{P}_{K}^{1}$ that maps the branch locus of $\bar{\pi}_{1, K}$ isomorphically onto that of $\bar{\pi}_{j, K}$. Furthermore, since $q \geq 2$, the branch locus of $\bar{\pi}_{i, K}$ consists of at least three points, implying that the $K$-automorphism $\bar{\theta}_{K}$ for which $\bar{\pi}_{j, K} \circ \Psi_{K}=\bar{\theta}_{K} \circ \bar{\pi}_{1, K}$ is unique, hence descends to a $\mathbb{C}(t)$-automorphism $\bar{\theta}$ such that $\bar{\pi}_{j} \circ \Psi=\bar{\theta} \circ \bar{\pi}_{1}$. Since $\bar{Y}_{i} \backslash Y_{i}$ consists of a unique $\mathbb{C}(t)$-rational point, say $\infty_{i}$, and since $\Psi\left(\infty_{1}\right)=\infty_{j}, \bar{\theta}$ restricts to an isomorphism $\theta: \mathbb{A}_{\mathbb{C}(t)}^{1}=\mathbb{P}_{\mathbb{C}(t)}^{1} \backslash \bar{\pi}_{1}\left(\infty_{1}\right) \stackrel{\sim}{\rightarrow} \mathbb{A}_{\mathbb{C}(t)}^{1}=\mathbb{P}_{\mathbb{C}(t)}^{1} \backslash \bar{\pi}_{j}\left(\infty_{j}\right)$ for which the diagram above commutes, as desired.

\section{On the case of negative logarithmic Kodaira dimension}

The aim of this section is to construct explicit examples of smooth affine varieties of negative logarithmic Kodaira dimension failing the Laurent Cancellation property. Let us first remark that such examples must be of dimension at least three. Indeed, we can extend a result of [3], that states that Laurent Cancellation does 
hold for smooth factorial affine surfaces of negative logarithmic Kodaira dimension, to the case of arbitrary smooth affine surfaces of negative logarithmic Kodaira dimension.

Theorem 13. Two smooth affine surfaces $S$ and $S^{\prime}$ of negative logarithmic Kodaira dimension have isomorphic $\mathbb{A}_{*}^{1}$-cylinders $S \times \mathbb{A}_{*}^{1}$ and $S^{\prime} \times \mathbb{A}_{*}^{1}$ if and only if they are isomorphic.

Proof. By [9], a smooth affine surface of negative logarithmic Kodaira dimension admits a faithfully flat morphism $\rho: S \rightarrow B$ over a smooth curve $B$, with generic fiber isomorphic to the affine line over the function field of $B$, called an $\mathbb{A}^{1}$-fibration. Thus $\Gamma\left(S, \mathcal{O}_{S}^{*}\right)=\rho^{*} \Gamma\left(B, \mathcal{O}_{B}^{*}\right)$ and combined with the fact that cancellation holds when all invertible functions on $S$ or $S^{\prime}$ are constant (see [5,3]), we can restrict from now on to the case where $S$ and $S^{\prime}$ admit $\mathbb{A}^{1}$-fibrations $\rho: S \rightarrow B$ and $\rho^{\prime}: S^{\prime} \rightarrow B^{\prime}$ over smooth curves $B$ and $B^{\prime}$ respectively, admitting non constant invertible functions. In particular $B$ and $B^{\prime}$ are affine, of nonnegative logarithmic Kodaira dimension, implying that every morphism from $\mathbb{A}^{1}$ to $B \times \mathbb{A}_{*}^{1}$ or $B^{\prime} \times \mathbb{A}_{*}^{1}$ is constant. Since all irreducible components of fibers of $\rho: S \rightarrow B$ over closed points of $B$ are isomorphic to $\mathbb{A}^{1}$, it follows that every isomorphism $\Psi: S \times \mathbb{A}_{*}^{1} \stackrel{\sim}{\rightarrow} S^{\prime} \times \mathbb{A}_{*}^{1}$ descends to an isomorphism $\psi: B \times \mathbb{A}_{*}^{1} \rightarrow B^{\prime} \times \mathbb{A}_{*}^{1}$ making the following diagram commutative

$$
\begin{array}{rll}
S \times \mathbb{A}_{*}^{1} \stackrel{\Psi}{\longrightarrow} & S^{\prime} \times \mathbb{A}_{*}^{1} \\
\pi=\left(\rho, \mathrm{pr}_{2}\right) \mid & & \downarrow \pi^{\prime}=\left(\rho^{\prime}, \mathrm{pr}_{2}\right) \\
B \times \mathbb{A}_{*}^{1} \stackrel{\psi}{\longrightarrow} & B^{\prime} \times \mathbb{A}_{*}^{1} .
\end{array}
$$

If either $\kappa(B) \neq 0$ or $\kappa\left(B^{\prime}\right) \neq 0$ then, by Iitaka-Fujita strong cancellation Theorem [7], $\psi$ descends further to an isomorphism $\bar{\psi}: B \rightarrow B^{\prime}$ such that $\operatorname{pr}_{B^{\prime}} \circ \psi=\bar{\psi} \circ \operatorname{pr}_{B}$. In the case where $\kappa(B)=\kappa\left(B^{\prime}\right)=0, B$ and $B^{\prime}$ are both isomorphic to $\mathbb{A}_{*}^{1}=\operatorname{Spec}\left(\mathbb{C}\left[u^{ \pm 1}\right]\right)$ and we have the following alternative: either $\rho: S \rightarrow B$ is a locally trivial, hence trivial, $\mathbb{A}^{1}$-bundle and then so is $\rho^{\prime}: S^{\prime} \rightarrow B^{\prime}$ by the commutativity of the above diagram or $\rho: S \rightarrow B$ has at least a degenerate fiber. In the second case, letting $b_{1}, \ldots, b_{s} \in B$ and $b_{1}^{\prime}, \ldots, b_{s^{\prime}}^{\prime} \in B^{\prime}$ be the closed points over which the fibers or $\rho$ and $\rho^{\prime}$ respectively are degenerate, the morphisms $\pi$ and $\pi^{\prime}$ degenerate respectively over the sections $\left\{b_{i}\right\} \times \mathbb{A}_{*}^{1}$ and $\left\{b_{i}^{\prime}\right\} \times \mathbb{A}_{*}^{1}$ of the projections $\operatorname{pr}_{\mathbb{A}_{*}^{1}}: B \times \mathbb{A}_{*}^{1} \rightarrow \mathbb{A}_{*}^{1}$ and $\operatorname{pr}_{\mathbb{A}_{*}^{1}}: B^{\prime} \times \mathbb{A}_{*}^{1} \rightarrow \mathbb{A}_{*}^{1}$. Identifying $B \times \mathbb{A}_{*}^{1}$ and $B^{\prime} \times \mathbb{A}_{*}^{1}$ with the torus $\mathbf{T}^{2}=\operatorname{Spec}\left(\mathbb{C}\left[u^{ \pm 1}, t^{ \pm 1}\right]\right)$, the automorphism $\psi$ has the form $(u, t) \mapsto\left(\lambda_{1} u^{\alpha} t^{\beta}, \lambda_{2} u^{\gamma} t^{\delta}\right)$ where $\lambda_{i} \in \mathbb{C}^{*}$ and $\left(\begin{array}{cc}\alpha & \beta \\ \gamma & \delta\end{array}\right) \in \mathrm{GL}_{2}(\mathbb{Z})$. The condition $\psi\left(\left\{b_{i}\right\} \times \mathbb{A}_{*}^{1}\right)=\left\{b_{j(i)}^{\prime}\right\} \times \mathbb{A}_{*}^{1}, i=1, \ldots, s, j(i) \in\left\{1, \ldots, s^{\prime}\right\}$ implies that $\beta=0$ hence that $\alpha \delta= \pm 1$. It follows that $\psi$ descends to an isomorphism $\bar{\psi}: B \rightarrow B^{\prime}$ of the form $u \mapsto \lambda_{1} u^{ \pm 1}$. Summing up, either $S$ and $S^{\prime}$ are both isomorphic to the trivial $\mathbb{A}^{1}$-bundle over $\mathbb{A}_{*}^{1}$ and we are done already, or we have a commutative diagram

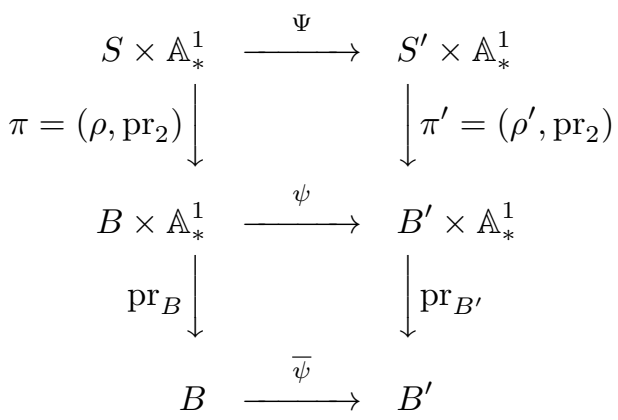

for some isomorphism $\bar{\psi}: B \stackrel{\sim}{\rightarrow} B^{\prime}$. Replacing $S^{\prime}$ by the isomorphic surface $S^{\prime} \times_{B^{\prime}} B$, we may assume further that $B^{\prime}=B$ and that $\bar{\psi}=\operatorname{id}_{B}$. The commutativity of the above diagram then implies that $\psi$ maps the section $C=B \times\{1\} \subset B \times \mathbb{A}_{*}^{1}$ isomorphically onto a section $C^{\prime} \subset B^{\prime} \times \mathbb{A}_{*}^{1}$ or $\operatorname{pr}_{B^{\prime}}$ while $\Psi$ maps $\pi^{-1}(C)$ isomorphically onto $\pi^{\prime-1}\left(C^{\prime}\right)$. The assertion follows since $\pi^{-1}(C)$ and $\pi^{\prime-1}\left(C^{\prime}\right)$ are isomorphic to $S$ and $S^{\prime}$ respectively.

From now on, we will use the following notation. 
Notation 14. Given integers $n \geq 1$ and $m \geq 2$, we denote by $f_{n, m}$ the polynomial

$$
f_{n, m}=\left(\prod_{i=1}^{n} x_{i}^{2}\right) z-y^{m} \in \mathbb{C}\left[x_{1}, \ldots, x_{n}\right][y, z] .
$$

Since $f_{n, m}$ is a semi-invariant of weight $m$ for the linear $\mathbb{G}_{m}$-action on $\mathbb{A}^{n+2}=\operatorname{Spec}\left(\mathbb{C}\left[x_{1}, \ldots, x_{n}\right][y, z]\right)$ with weights $(1, \ldots, 1,1, m-2 n)$, we will follow the notation of the previous sections and consider, for every $\ell \in \mathbb{Z}_{\geq 1}$, the hypersurface $X_{n, m, \ell}=\widetilde{X}_{f_{n, m}, \ell}$ of $\mathbb{A}^{n+3}=\operatorname{Spec}\left(\mathbb{C}\left[x_{1}, \ldots, x_{n}\right][y, z, t]\right)$ defined by the equation

$$
t^{\ell}\left(x_{1}^{2} \cdots x_{n}^{2} z-y^{m}\right)=1 .
$$

Lemma 15. Every hypersurface $X_{n, m, \ell}$ defined above is smooth and has negative logarithmic Kodaira dimension. Moreover, $X_{n, m, \ell}$ is factorial provided that $\ell$ is relatively prime with $m$.

Proof. Consider the open subset $\mathcal{U}=X_{n, m, \ell} \backslash\left\{x_{1} \cdots x_{n}=0\right\}$ of $X_{n, m, \ell}$. Since

$$
\mathcal{U}=\left\{\left(x_{1}, \ldots, x_{n}, y, z, t\right) \in \mathbb{A}^{n+3} \mid x_{1} \cdots x_{n} t \neq 0, z=x_{1}^{-2} \cdots x_{n}^{-2}\left(t^{-\ell}+y^{m}\right)\right\} \simeq\left(\mathbb{A}_{*}^{1}\right)^{n+1} \times \mathbb{A}^{1},
$$

it follows that $\mathcal{U}$ has negative logarithmic Kodaira dimension, hence that $X_{n, m, \ell}$ has negative logarithmic Kodaira dimension too.

From now on, we suppose that $\ell$ is coprime with $m$. Then, since

$$
\mathcal{O}\left(X_{n, m, \ell}\right) /\left(x_{n}\right) \simeq \mathbb{C}\left[x_{1}, \ldots, x_{n-1}, y, z, t\right] /\left(t^{l} y^{m}+1\right)
$$

is an integral domain, when $\ell$ is coprime with $m$, it follows that $\left(x_{n}\right)$ is a prime ideal of $\mathcal{O}\left(X_{n, m, \ell}\right)$. We further consider the localization $\mathcal{O}\left(X_{n, m, \ell}\right)_{x_{n}}$ of $\mathcal{O}\left(X_{n, m, \ell}\right)$ with respect to this ideal $\left(x_{n}\right)$. If $n=1$, we can express $z=x_{1}^{-2}\left(y^{m}+t^{-\ell}\right)$ and we thus get that $\mathcal{O}\left(X_{n, m, \ell}\right)_{x_{1}} \simeq \mathbb{C}\left[y, x_{1}^{ \pm 1}, t^{ \pm 1}\right]$ is factorial. By a well-known result of Nagata (see e.g. [12]), this implies that $\mathcal{O}\left(X_{n, m, \ell}\right)$ is factorial for $n=1$. By induction on $n$, it follows that

$$
\mathcal{O}\left(X_{n, m, \ell}\right)_{x_{n}} \simeq \mathbb{C}\left[x_{n}^{ \pm 1}\right]\left[x_{1}, \ldots, x_{n-1}, y, z, t\right] /\left(t^{\ell}\left(\prod_{i=1}^{n-1} x_{i}^{2} z-y^{m}\right)-1\right)
$$

is factorial for all $n \geq 1$, and so Nagata's result allows us to conclude that $\mathcal{O}\left(X_{n, m, \ell}\right)$ is factorial.

The next theorem is the main result of this section. It shows that we can find counterexamples to the Laurent Cancellation Problem among the above varieties $X_{n, m, \ell}$.

Theorem 16. Let $n \geq 1, m \geq 2$ and let $\ell, \ell^{\prime} \geq 1$ relatively prime with $m$ be such that $\ell^{\prime}$ is not congruent to $\pm \ell$ modulo $m$. Then the hypersurfaces

$$
X_{n, m, \ell}=\left\{t^{\ell}\left(x_{1}^{2} \cdots x_{n}^{2} z-y^{m}\right)=1\right\} \subset \mathbb{A}^{n+3}
$$

and

$$
X_{n, m, \ell^{\prime}}=\left\{t^{\ell^{\prime}}\left(x_{1}^{2} \cdots x_{n}^{2} z-y^{m}\right)=1\right\}
$$

are nonisomorphic factorial affine varieties of dimension $d=n+2$ with isomorphic $\mathbb{A}_{*}^{1}$-cylinders

$$
X_{n, m, \ell} \times \mathbb{A}_{*}^{1} \simeq X_{n, m, \ell^{\prime}} \times \mathbb{A}_{*}^{1} .
$$

Proof. On the one hand, the $\mathbb{A}_{*}^{1}$-cylinders $X_{n, m, \ell} \times \mathbb{A}_{*}^{1}$ and $X_{n, m, \ell^{\prime}} \times \mathbb{A}_{*}^{1}$ are isomorphic by Proposition 2. On the other hand, the first assertion of the theorem follows directly from Lemma 8 and Proposition 17 below. Indeed, there exists an element $\alpha \in \mathbf{k}=\mathbb{C}(t)$ such that $t^{-\ell}=\alpha^{m} t^{-\left( \pm \ell^{\prime}\right)}$ if and only if $\ell$ is congruent to $\pm \ell^{\prime}$ modulo $m$.

Proposition 17. Let $n \geq 1$ and $m \geq 2$ be integers and let $\mathbf{k}$ be a field of characteristic zero. Consider, for every $\lambda \in \mathbf{k}$, the ring

$$
B_{\lambda}=\mathbf{k}\left[x_{1}, \ldots, x_{n}, y, z\right] /\left(x_{1}^{2} \cdots x_{n}^{2} z-y^{m}-\lambda\right) .
$$

Two such rings $B_{\lambda}$ and $B_{\lambda^{\prime}}$ are isomorphic if and only if there exists a constant $\alpha \in \mathbf{k}^{*}$ such that $\lambda=\alpha^{m} \lambda^{\prime}$.

Proof. If $\lambda=\alpha^{m} \lambda^{\prime}$ for some $\alpha \in \mathbf{k}^{*}$, then it is obvious that $B_{\lambda}$ and $B_{\lambda^{\prime}}$ are isomorphic via a linear change of coordinates. It remains to prove the converse implication. For this, we use techniques from the theory of locally nilpotent derivations, that were mainly developed by Makar-Limanov and became progressively classical tools in affine algebraic geometry. Actually, our proof simply recollects arguments that were already given in [2] and [11]. 
Let $\delta$ be a nonzero locally nilpotent derivations on $B_{\lambda}$. From [2, Proposition 2.2 and Corollary 2.1], which remain valid over any field of characteristic zero, we have that $\operatorname{Ker}(\delta)=\mathbb{C}\left[x_{1}, \ldots, x_{n}\right]$ and $\operatorname{Ker}\left(\delta^{2}\right) \subset$ $\mathbb{C}\left[x_{1}, \ldots, x_{n}, y\right]$ both hold. Then, arguing exactly as in [11, Proposition 2.3], it follows that $\operatorname{Ker}\left(\delta^{2}\right)=$ $\mathbb{C}\left[x_{1}, \ldots, x_{n}\right] y+\mathbb{C}\left[x_{1}, \ldots, x_{n}\right]$ and that

$$
\delta=h\left(x_{1}, \ldots, x_{n}\right)\left(x_{1}^{2} \cdots x_{n}^{2} \frac{\partial}{\partial y}+m y^{m-1} \frac{\partial}{\partial z}\right),
$$

for some $h\left(x_{1}, \ldots, x_{n}\right) \in \mathbf{k}\left[x_{1}, \ldots, x_{n}\right]$.

Now, let $\varphi: B_{\lambda} \rightarrow B_{\lambda^{\prime}}$ be an isomorphism and denote by $x_{1}, \ldots, x_{n}, y, z$ (resp. $\left.x_{1}^{\prime}, \ldots, x_{n}^{\prime}, y^{\prime}, z^{\prime}\right)$ the images of $x_{1}, \ldots, x_{n}, y, z$ in $B_{\lambda}$ (resp. in $B_{\lambda^{\prime}}$ ). Similarly as in [11, Proposition 2.5] we can infer from the above properties that there exist nonzero constants $a_{1}, \ldots, a_{n} \in \mathbf{k}^{*}, \alpha \in \mathbf{k}^{*}$, a polynomial $\beta \in \mathbf{k}\left[X_{1}, \ldots, X_{n}\right]$ and a bijection $\sigma$ of the set $\{1, \ldots, n\}$ such that $\varphi\left(x_{i}\right)=a_{i} \varphi\left(x_{\sigma(i)}^{\prime}\right)$ for all $1 \leq i \leq n$ and such that $\varphi(y)=\alpha y^{\prime}+\beta\left(x_{1}^{\prime}, \ldots, x_{n}^{\prime}\right)$.

Finally, we write that

$$
\varphi\left(x_{1}^{2} \cdots x_{n}^{2} z-y^{m}-\lambda\right)=\left(\prod_{i=1}^{n} a_{i}^{2}\right)\left(x_{1}^{\prime} \cdots x_{n}^{\prime}\right)^{2} \varphi(z)-\left(\alpha y^{\prime}+\beta\left(x_{1}^{\prime}, \ldots, x_{n}^{\prime}\right)\right)^{m}-\lambda
$$

is equal to zero in $B_{\lambda^{\prime}}$. This gives the existence of polynomials $R$ and $S$ in $\mathbf{k}\left[X_{1}, \ldots, X_{n}, Y, Z\right]$ such that

$$
\left(\prod_{i=1}^{n} a_{i}^{2}\right)\left(X_{1} \cdots X_{n}\right)^{2} R-\left(\alpha Y+\beta\left(X_{1}, \ldots, X_{n}\right)\right)^{m}-\lambda=S \cdot\left(X_{1}^{2} \cdots X_{n}^{2} Z-Y^{m}-\lambda^{\prime}\right) .
$$

From this, we deduce that

$$
-(\alpha Y+\beta(0, \ldots, 0))^{m}-\lambda=S(0, \ldots, 0, Y, 0) \cdot\left(-Y^{m}-\lambda^{\prime}\right),
$$

which implies that $S(0, \ldots, 0, Y, 0)$ is in fact a nonzero constant. Therefore, we have $\beta(0, \ldots, 0)=0$, $S(0, \ldots, 0, Y, 0)=\alpha^{m}$ and thus $\lambda=S(0, \ldots, 0, Y, 0) \lambda^{\prime}=\alpha^{m} \lambda^{\prime}$, as desired.

Example 18. The simplest case in Theorem 16 holds when $n=1, m=5, \ell=1$ and $\ell^{\prime}=2$, in which case we obtain that the factorial threefolds in $\mathbb{A}^{4}=\operatorname{Spec}(\mathbb{C}[x, y, z, t])$ defined by the equations $t\left(x^{2} z-y^{5}\right)=1$ and $t^{2}\left(x^{2} z-y^{5}\right)=1$, respectively, are nonisomorphic but have isomorphic $\mathbb{A}_{*}^{1}$-cylinders.

\section{Affine varieties With NONisomorphic SQUare Roots}

In this section, we construct nonisomorphic affine algebraic varieties $X$ and $Y$ with isomorphic cartesian products $X \times X$ and $Y \times Y$. To begin with, we adapt Proposition 2 to obtain isomorphic cartesian products.

Lemma 19. Let $n \geq 1$ and let $f$ (resp. $g$ ) be a regular function on the affine $n$-space $X=\mathbb{A}^{n}$ which is semiinvariant of weight $m \neq 0$ for some action $\mu: \mathbb{G}_{m} \times X \rightarrow X$ (resp. $\nu: \mathbb{G}_{m} \times X \rightarrow X$ ) of the multiplicative group on $X$. Let $a, b \geq 1$ be integers such that ab is congruent to 1 modulo $m^{2}$. Then the products $\widetilde{X}_{f, \ell} \times \widetilde{X}_{g, \ell^{\prime}}$ and $\widetilde{X}_{f, a \ell} \times \widetilde{X}_{g, b \ell^{\prime}}$ are isomorphic varieties for all integers $\ell, \ell^{\prime} \geq 1$.

Proof. Let us identify the products $\Pi_{1}=\widetilde{X}_{f, \ell} \times \widetilde{X}_{g, \ell^{\prime}}$ and $\Pi_{2}=\widetilde{X}_{f, a \ell} \times \widetilde{X}_{g, b \ell^{\prime}}$ with the closed subvarieties of $\left(X \times \mathbb{A}_{*}^{1}\right)^{2}=\operatorname{Spec}\left(\mathbb{C}\left[x_{1} \ldots, x_{n}, y_{1}, \ldots, y_{n}\right]\left[t^{ \pm 1}, s^{ \pm 1}\right]\right)$ defined by the ideals

$$
I_{1}=\left(t^{\ell} f\left(x_{1}, \ldots, x_{n}\right)-1, s^{\ell^{\prime}} g\left(y_{1}, \ldots, y_{n}\right)-1\right)
$$

and

respectively.

$$
I_{2}=\left(t^{a \ell} f\left(x_{1}, \ldots, x_{n}\right)-1, s^{b \ell^{\prime}} g\left(y_{1}, \ldots, y_{n}\right)-1\right)
$$

Since $a b$ is congruent to 1 modulo $m^{2}$, there exists an integer $c \in \mathbb{Z}$ such that the matrix $\left(\begin{array}{cc}a & c m \\ m & b\end{array}\right)$ belongs to $\mathrm{SL}_{2}(\mathbb{Z})$. This matrix corresponds to an automorphism $\sigma(t, s)=\left(t^{a} s^{c m}, t^{m} s^{b}\right)$ of the torus $\left(\mathbb{A}_{*}^{1}\right)^{2}=$ $\operatorname{Spec}\left(\mathbb{C}\left[t^{ \pm 1}, s^{ \pm 1}\right]\right)$. Then, it suffices to remark that the automorphism of $\left(X \times \mathbb{A}_{*}^{1}\right)^{2}$ defined by $(x, y, t, s) \mapsto$ $\left(\mu\left(s^{-c \ell}, x\right), \nu\left(t^{-\ell^{\prime}}, y\right), \sigma(t, s)\right)$ maps $\Pi_{2}$ isomorphically onto $\Pi_{1}$. 
Corollary 20. Let $X=\mathbb{A}^{n}$ and let $f \in \mathcal{O}(X)$ be semi-invariant of weight $m \neq 0$ for a $\mathbb{G}_{m}$-action on $X$. Suppose that there exist integers $a, b \geq 1$ satisfying the two following congruences

$$
a+b \equiv 0 \quad \bmod (m) \quad \text { and } \quad a b \equiv 1 \quad \bmod \left(m^{2}\right) .
$$

Then, the varieties $\tilde{X}_{f, 1} \times \widetilde{X}_{f, 1}$ and $\tilde{X}_{f, a} \times \tilde{X}_{f, a}$ are isomorphic.

Proof. On one hand, we have that $\tilde{X}_{f, 1} \times \tilde{X}_{f, 1}$ is isomorphic to $\tilde{X}_{f, a} \times \widetilde{X}_{f, b}$ by Lemma 19. On the other hand, it follows from Proposition 2 that $\widetilde{X}_{f, a}$ and $\widetilde{X}_{f, b}$ are isomorphic.

Combining the above corollary with the results of the previous sections, we obtain examples of nonisomorphic affine varieties whose square are isomorphic. The simplest case occurs for $m=5$. Let us denote, as in Theorem 16, by $X_{n, m, \ell}$ the hypersurface of $\mathbb{A}^{n+3}$ defined by the equation $t^{\ell}\left(x_{1}^{2} \cdots x_{n}^{2} z-y^{m}\right)=1$.

Proposition 21. The varieties $X=X_{n, 5,1}$ and $Y=X_{n, 5,2}$ are not isomorphic, although their squares $X \times X$ and $Y \times Y$ are isomorphic.

Proof. We deduce that $X \times X$ and $Y \times Y$ are isomorphic from Corollary 20 with $m=5, a=2$ and $b=13$. The fact that $X$ and $Y$ are not isomorphic is a particular case of Theorem 16.

Corollary 20 allows us to find also two-dimensional examples. In particular, the two nonisomorphic surfaces of Proposition 10 have isomorphic squares.

Proposition 22. Let $X_{1}, X_{3} \subset \mathbb{A}^{3}=\operatorname{Spec}(\mathbb{C}[x, y, t])$ be the (nonisomorphic by Proposition 10) hypersurfaces defined by the equation $t\left(x^{2}+y^{5}\right)=1$ and $t^{3}\left(x^{2}+y^{5}\right)=1$, respectively. Then, $X_{1} \times X_{1}$ and $X_{3} \times X_{3}$ are isomorphic.

Proof. It suffices to apply Corollary 20 with $m=10, a=3$ and $b=67$.

Finally, let us remark that such phenomenon cannot occur for affine curves.

Proposition 23. Two smooth complex affine curves $C_{1}$ and $C_{2}$ have isomorphic squares $C_{1} \times C_{1} \simeq C_{2} \times C_{2}$ if and only if they are isomorphic.

Proof. Suppose that there exists an isomorphism $\varphi: C_{1} \times C_{1} \stackrel{\sim}{\rightarrow} C_{2} \times C_{2}$. Then the restriction of the first projection $\operatorname{pr}_{1}: C_{2} \times C_{2} \rightarrow C_{2}$ to the image by $\varphi$ of a fiber of the first or the second projection $\operatorname{pr}_{i}: C_{1} \times C_{1} \rightarrow C_{1}, i=1,2$, defines a dominant morphism $\pi_{2}: C_{1} \rightarrow C_{2}$. Exchanging the roles of $C_{1}$ and $C_{2}$, we obtain in a similar way a dominant morphism $\pi_{1}: C_{2} \rightarrow C_{1}$. These extend to finite morphisms $\bar{\pi}_{i}: \bar{C}_{j} \rightarrow \bar{C}_{i}$ between the smooth projective models of $C_{1}$ and $C_{2}$, and we deduce from Riemann-Hurwitz formula that $\bar{C}_{1}$ and $\bar{C}_{2}$ have the same genus $g$. Since $C_{i}$ is affine, $\bar{C}_{i} \backslash C_{i}$ is non-empty, consisting of a finite number of points $p_{i, j}, j=1, \ldots, r_{i}, i=1,2$. By Kunneth formula,

$$
H_{2}\left(C_{i} \times C_{i} ; \mathbb{Z}\right) \simeq H_{1}\left(C_{i} ; \mathbb{Z}\right) \otimes H_{1}\left(C_{i} ; \mathbb{Z}\right) \simeq \mathbb{Z}^{2 g+r_{i}-1} \otimes \mathbb{Z}^{2 g+r_{i}-1},
$$

where we have used that $H_{2}\left(C_{i} ; \mathbb{Z}\right)=0$ because $C_{i}$ is affine. Thus $r_{1}=r_{2}=r \geq 1$.

If $g \geq 2$ then Riemann-Hurwitz formula actually implies that $\bar{\pi}_{1}$ and $\bar{\pi}_{2}$ are isomorphisms. Thus $\pi_{2}$ : $C_{1} \rightarrow C_{2}$ and $\pi_{1}: C_{2} \rightarrow C_{1}$ are both open immersions and are both surjective for otherwise $\pi_{1} \circ \pi_{2}$ would be a strict open embedding of $C_{1}$ into itself, which is impossible. So $\pi_{1}: C_{2} \rightarrow C_{1}$ and $\pi_{2}$ are isomorphisms by virtue of Zariski Main Theorem.

If $g=1$, then by Riemann-Hurwitz formula again, $\bar{\pi}_{1}: \bar{C}_{2} \rightarrow \bar{C}_{1}$ is a finite unramified morphism, say of degree $d \geq 1$. Since $\bar{\pi}_{1}$ is the extension of a morphism $\pi_{1}: C_{2} \rightarrow C_{1}, \bar{\pi}_{1}^{-1}\left(\bar{C}_{1} \backslash C_{1}\right) \subset \bar{C}_{2} \backslash C_{2}$ and so $d r=d \cdot \sharp\left(\bar{C}_{1} \backslash C_{1}\right) \leq \sharp\left(\bar{C}_{2} \backslash C_{2}\right)=r$. Thus $d=1$, and the conclusion follows from the same argument as above.

If $g=0$, then $\bar{\pi}_{1}: \bar{C}_{2} \simeq \mathbb{P}^{1} \rightarrow \bar{C}_{1} \simeq \mathbb{P}^{1}$ is a finite morphism of degree $d \geq 1$. If $d=1$, then by the same argument again, $\pi_{1}: C_{2} \rightarrow C_{1}$ is an isomorphism. Otherwise, if $d>1$, then since $\bar{\pi}_{1}^{-1}\left(\bar{C}_{1} \backslash C_{1}\right) \subset \bar{C}_{2} \backslash C_{2}$ and $\sharp\left(\bar{C}_{1} \backslash C_{1}\right)=\sharp\left(\bar{C}_{2} \backslash C_{2}\right)=r \geq 1$, it must be that $\bar{\pi}_{1}$ is totally ramified over every point of $\bar{C}_{1} \backslash C_{1}$, with $\bar{\pi}_{1}\left(\bar{C}_{2} \backslash C_{2}\right)=\bar{C}_{1} \backslash C_{1}$. By Riemann-Hurwitz formula, we have

$$
2=2 d-\sum_{p \in \overline{C_{1} \backslash C_{1}}}(d-1)-\delta=2 d-r(d-1)-\delta
$$

for some $\delta \geq 0$. Rewriting this equality in the form $(d-1)(2-r)=\delta \geq 0$, we conclude that either $r=1$, in which case $C_{1} \simeq C_{2} \simeq \mathbb{A}^{1}$, or $r=2$ and then $C_{1} \simeq C_{2} \simeq \mathbb{A}_{*}^{1}$. 


\section{REFERENCES}

[1] S. M. Bhatwadekar and N. Gupta, The structure of a Laurent polynomial fibration in n variables, J. Algebra 353 (2012), $142-157$.

[2] A. Dubouloz, Additive group actions on Danielewski varieties and the cancellation problem., Math. Z. 255 (2007), no. 1, $77-93$.

[3] A. Dubouloz, On the cancellation problem for algebraic tori, Preprint arXiv:1412.2213 [math.AG], to appear in Ann. Inst. Fourier (Grenoble).

[4] T. J. Ford, The group of units on an affine variety, J. Algebra Appl. 13 (2014), no. 8, 1450065, 27 pp.

[5] G. Freudenburg, Laurent cancellation for rings of transcendence degree one over a field, Automorphisms in Birational and Affine Geometry, Springer Proceedings in Mathematics \& Statistics, Volume 79, (2014), 313-326.

[6] G. Gonzalez-Diez, On Prime Galois Coverings of the Riemann Sphere,Ann. Mat. Pura Appl. 168 (1995), 1-15.

[7] S. Iitaka and T. Fujita, Cancellation theorem for algebraic varieties., J. Fac. Sci., Univ. Tokyo, Sect. IA Math. 24 (1977), $123-127$.

[8] M. Miyanishi, Open algebraic surfaces, CRM Monogr. Ser., 12, Amer. Math. Soc., Providence, RI, 2001.

[9] M. Miyanishi and T. Sugie, Affine surfaces containing cylinderlike open sets, J. Math. Kyoto Univ. 20 (1980), no. 1, 11-42.

[10] N. Nakajo, On the equivalence problem of cyclic branched covering of the Riemann sphere, Kyushu J. Math. 53 (1999), $127-131$.

[11] P.-M. Poloni, A note on the stable equivalence problem, J. Math. Soc. Japan. 67 (2015), no. 2, $753-761$.

[12] P. Samuel, Lectures on unique factorization domains. (Notes by M. Pavman Murthy) Tata Institute of Fundamental Research Lectures on Mathematics, No. 30, Bombay, (1964).

Adrien Dubouloz, imb UMr5584, CnRs, Univ. Bourgogne Franche-Comté, F-21000 Dijon, France.

E-mail address: adrien.dubouloz@u-bourgogne.fr

Pierre-Marie Poloni, Universität Bern, Mathematisches Institut, Sidlerstrasse 5, Ch-3012 Bern, SwitzerLAND,

E-mail address: pierre.poloni@math.unibe.ch 